\title{
Fuzzy Scaling Analysis of a Mouse Mutant With Brain Morphological Changes
}

\author{
Tuan D. Pham, Senior Member, IEEE, Catharina C. Müller, and Denis I. Crane
}

\begin{abstract}
Scaling behavior inherently exists in fundamental biological structures, and the measure of such an attribute can only be known at a given scale of observation. Thus, the properties of fractals and power-law scaling have become attractive for research in biology and medicine because of their potential for discovering patterns and characteristics of complex biological morphologies. Despite the successful applications of fractals for the life sciences, the quantitative measure of the scale invariance expressed by fractal dimensions is limited in more complex situations, such as for histopathological analysis of tissue changes in disease. In this paper, we introduce the concept of fuzzy scaling and its analysis of a mouse mutant with postnatal brain morphological changes.
\end{abstract}

Index Terms-Fractals, fuzzy $c$-means (FCMs), fuzzy sets (FCMs), mouse brains, peroxisomal dysfunction, scaling, Zellweger syndrome.

\section{INTRODUCTION}

$\mathbf{R}$ APID advancements in biology and medicine have created a demand for advanced computerized tools and information science techniques to accelerate our understanding of disease processes at the cell and tissue levels, with the ultimate goal of improved disease treatments. In terms of image analysis, a persistent problem in the histopathological analysis of disease is the high variation in reported observations, both within and between analyses. This kind of problem is often addressed by the use of medical expert systems embedded with image processing techniques. Accordingly, there is an ongoing need for better computer-based methods for pathological studies to provide both improved practical diagnostic assistance to enhance artificial-intelligence-based diagnosis and further insight into disease processes [1].

In this study, we have addressed this problem through the analysis of a mouse model of a human neurodegenerative disease, Zellweger syndrome, which manifests with severe brain morphological and functional abnormalities. Zellweger syndrome results from defective biogenesis of the peroxisome, which is a ubiquitous cellular organelle [2]. Mutations in proteins required for peroxisome biogenesis, or peroxins, disrupt the molecular apparatus required for posttranslational import of

Manuscript received October 29, 2007; revised September 24, 2008. First published April 14, 2009; current version published July 6, 2009. This work was supported by the Australian Research Council's Discovery Projects Funding Scheme under Project DP0877414.

T. D. Pham is with the School of Information Technology and Electrical Engineering, The University of New South Wales, Canberra, A.C.T. 2600, Australia (e-mail: t.pham@adfa.edu.au).

C. C. Müller and D. I. Crane are with the School of Biomolecular and Physical Sciences, and Eskitis Institute for Cell and Molecular Therapies, Griffith University, Nathan, Qld. 4111, Australia (e-mail: c.muller. @ griffith. edu.au; d.crane@griffith.edu.au).

Color versions of one or more of the figures in this paper are available online at http://ieeexplore.ieee.org.

Digital Object Identifier 10.1109/TITB.2009.2019638 content proteins of the peroxisome, leading to the absence, or deficiency, of peroxisomal metabolic capacity [3].

To generate a mouse model of this disorder, we initially developed a knockout mutant deficient in the peroxin Pex13, a peroxisomal membrane protein demonstrated to be required for import of peroxisome proteins in humans [4]-[6]. Pex 13 knockout mice display a phenotype that closely resembles the human disease, including neonatal death [7]. In more recent study, we have expanded the range of mouse mutants to include one that has brain-specific deficiency of Pex13 (Nguyen, Müller, and Crane, unpublished data). This mutant has allowed analyses of brain postnatal changes that are not possible in the knockout mutant due to its neonatal lethality. In the study, we focus on morphological changes apparent in the cerebellum of Pex13 brain mutants at various postnatal stages. Manual analysis and quantification of changes in microscopic images of brains of the mutant mice are a cumbersome task. We were, therefore, interested in developing a more automated process for establishing patterns of change in brain morphology. For these purposes, we propose an approach that utilizes the conceptual framework of fuzzy scaling and its fuzzy fractal dimensions (FFDs).

Earlier studies on the applications of fractal analysis of pathological changes have been associated with cell morphology, neurons, tumor shape, retinal vessels, spread of viruses, and the modeling of periodontal diseases [8]-[11], and in which fractals were used to quantify geometrical changes in complex patterns. As an important additional example, the application of scaling power has demonstrated that the fractal dimensions for tumor vasculature are consistent and can be used for characterizing the mechanisms of vascular growth in tumors [12]. More recently, fractals have been utilized to study the cellular and microanatomical structures, including the distribution of bronchial capillaries, around large airways [13]. Again, because of the difficulty in quantifying the changes in the actin cytoskeleton of cardiac fibroblasts, cells critical for proper heart function, fractal dimensions have been used as a descriptor for quantifying the morphological changes in these cells responding to mechanical stress [14].

Although the results obtained from fractal analysis for quantifying irregular morphologies are promising and the notion of fractals has become an important concept for image analysis in pathology, several problems still remain in applying this method for quantitative analysis of biological systems. For example, the decision to perform fractal analysis on a certain location of a pathological sample, and the mixture of irregular and regular regions lead to a more difficult analysis [8]. Furthermore, the use of fractal dimensions may not always be an effective descriptor in the analysis of the spatial complexity of image features and image textural information. 
As an innovative approach, and to advance the scaling concept of fractals for characterizing the mouse brain mutants described before, we present in this paper a novel concept of fuzzy scaling and its derived FFDs. The remainder of this paper is organized as follows. Section II introduces the concept of fuzzy scaling. Section III discusses how FFDs can be used for pattern classification. Section IV presents some experimental results and discussion. Finally, the conclusion of the new research work is given in Section V.

\section{FUZZY SCALING}

The fractal dimension [15] is a mathematical expression of the space-filling properties of an object whose concept leads to many different types of fractal dimensions [16]. The simplest form of fractal dimensions is the self-similarity dimension. The self-similarities of the line, square, and cube are equal to 1,2, and 3, respectively. Consider a geometrically self-similar fractal object that consists of line segments. If each line segment is divided into $M$ smaller line segments, then $N$ smaller objects are produced. Furthermore, if the object is geometrically selfsimilar, then each of the objects of smaller sizes is an exact but reduced size copy of the whole object. The self-similarity dimension $d$ is then expressed as [16]

$$
N=M^{d}
$$

which can be written in another form as

$$
d=\frac{\log (N)}{\log (M)} .
$$

Because the self-similarity dimension requires that each smaller subject formed by the division of the whole object must be an exact copy of the whole object, it can only be used to study objects that are geometrically self-similar. Such a fractal dimension is not very useful for analyzing many real objects that usually have irregular shapes. Thus, more general fractal dimensions have been developed as more general forms of the fractal dimension. Two such popular forms are known as the capacity and the Hausdorff dimensions. The capacity of an object can be determined by covering it with balls of a radius $r$. The smallest number of balls $N(r)$ that covers all the parts of the object is counted. Then, the radius of the previous balls is reduced and again $N(r)$ is counted. The capacity is the value of $\log N(r) / \log (1 / r)$ in the limit as $r$ shrinks to 0 . The relationship of the capacity and the self-similarity dimensions is that if $M=1 / r$, then $N=M^{d}$. The Hausdorff-Besicovitch dimension is similar, but not identical, to the capacity dimension. In the capacity dimension, the object is covered with the number of balls $N(r)$ of a given radius $r$; whereas in the Hausdorff dimension, the object is covered with sets.

There are two general principles of the concept of the fractal dimension: the scaling dimension and the Hausdorff dimension [17]. The first principle, which is often called the telescopemicroscope principle, states that the decrease of the measuring scale of an object by a factor $s$ is equivalent to the increase of the measuring scale of the object by the factor $s$. The second principle states that any measurement of the "mass" of a fractal of the Hausdorff dimension $d$ contained in a box of side $s$ is proportional to $s^{d}$. There are many natural ways for computing the mass of a fractal set such as the box-counting method [16], the area-perimeter method [18], and the mass-radius method [15]. We develop herein a new method for obtaining the fractal dimension using the framework of the fuzzy $c$-means (FCMs) algorithm, which can be useful for extracting some novel feature of mass spectrometry data and easily implemented by the vector quantization (VQ) technique for pattern classification.

Let $M_{f c}$ be the fuzzy $c$-partition space and $J: M_{f c} \times \mathcal{R}^{c p} \rightarrow$ $\mathcal{R}^{+}$be [19]

$$
J_{m}(\mathbf{U}, \mathbf{v})=\sum_{k=1}^{n} \sum_{i=1}^{c}\left(u_{i k}\right)^{m}\left(d_{i k}\right)^{2}
$$

where $\mathbf{U} \in M_{f c}$ is a fuzzy partition of $\mathbf{X}=\left(\mathbf{x}_{1}, \mathbf{x}_{1}, \ldots, \mathbf{x}_{n}\right)$, $\mathbf{v}=\left(\mathbf{v}_{1}, \mathbf{v}_{2}, \ldots, \mathbf{v}_{c}\right) \in \mathcal{R}^{c p}$ with $\mathbf{v}_{i} \in \mathcal{R}^{p}$ is the cluster center of $u_{i}, 1 \leq i \leq c, d_{i k}=\left\|\mathbf{x}_{k}-\mathbf{v}_{i}\right\|$, where $\|\cdot\|$ is any inner product induced norm on $\mathcal{R}^{p}$, and $m \in[1, \infty)$.

Observation of (3) reveals that $d_{i k}$ is the measure of dissimilarity between each data point $\mathbf{x}_{k}$ and fuzzy cluster center $\mathbf{v}_{i}$, whose squared distance is then weighted by the term $\left(u_{i k}\right)^{m}$. Thus, $J_{m}$ is a square error clustering function, and solutions for this fuzzy clustering algorithm is to

$$
\underset{M_{f c} \times \mathcal{R}^{c p}}{\operatorname{minimize}} J_{m}(\mathbf{U}, \mathbf{v}) .
$$

There exists an infinite family of fuzzy clustering algorithms —one for each $m \in[1, \infty)$-via the conditions for solutions of (4).

In the FCMs algorithm, $c$, i.e., the number of clusters, needs to be given. In many practical cases, $c$ is unknown. It is reasonable to expect cluster substructure at more than one value of $c$, and therefore, it is necessary to estimate the most plausible value of $c$ for the cluster analysis. This problem is known as cluster validity. It is very difficult to formulate the cluster validity problem in a mathematically tractable manner, because the basic question is imposed on the definition of a cluster. For fuzzy clustering, one should examine which pairs of fuzzy groups/classes overlap, and this leads to the question of how fuzzy a fuzzy $c$-partition is. A heuristic solution to this problem is to calculate the measure of fuzziness in $\mathbf{U}$, and then assign $c$ as the most valid value that has the least fuzzy partitions.

The first functional designed for cluster validity measure is the partition coefficient [19]. This partition coefficient of a fuzzy $c$-partition of $\mathbf{U} \in M_{f c}$ of $\mathbf{X}$ is expressed as

$$
F(c)=\frac{1}{n} \sum_{k=1}^{n} \sum_{i=1}^{c}\left(u_{i k}\right)^{2} .
$$

Another equivalent expression for (5) that emphasizes various properties of $F$ is the Euclidean inner product for two matrices $\mathbf{I}, \mathbf{J} \in \mathbf{V}_{c n}$ is $\left\langle\langle\mathbf{I}, \mathbf{J}\rangle=\operatorname{Tr}\left(\mathbf{I} \mathbf{J}^{\mathrm{T}}\right)\right.$, where $\operatorname{Tr}$ is the trace of a matrix and $\mathbf{J}^{\mathrm{T}}$ is the transpose of $\mathbf{J}$. Equation (5) has alternative forms

$$
F(c)=\frac{\operatorname{Tr}\left(\mathbf{U} \mathbf{U}^{\mathrm{T}}\right)}{n}=\frac{\langle\mathbf{U}, \mathbf{U}\rangle}{n}=\frac{\|\mathbf{U}\|^{2}}{n} .
$$


Now it can be analyzed that if $F(c)=1$, then $\mathbf{U}$ contains no fuzzy clusters ( $\mathbf{U}$ consists of only zeros and ones); if $F(c)=1 / c$ (all elements in $\mathbf{U}$ is equal to $1 / c$ ), then $\mathbf{U}$ is completely fuzzy; and in general, $1 / c \leq F(c) \leq 1$. As $F(c)$ increases, the partition of the datasets is more effective. Thus, the formal strategy for selecting the most valid $c^{*}$ is as follows. Let $\Omega_{c}$ represents any finite set of optimal U's $\in M_{f c}$ and $c=2,3, \ldots, n-1$. The optimal $c^{*}$ is determined by direct search

$$
c^{*}=\arg \max _{c}\left[\max _{\Omega_{c}} F(c)\right] .
$$

An observation of the relationship between $c$ and $F(c)$ reveals that $F(c)$ can be interpreted as a type of the measure of the fuzziness or the "fuzzy mass" of the partition space $\mathbf{U}$ as a function of the number of clusters of size $c$. Based on the second principle of dimension for fractals, $F(c)$ is equivalent to the approximate power law

$$
F(c) \propto c^{d_{\mathrm{fcm}}}
$$

where $d_{\mathrm{fcm}}$ is called the FCM-based fractal dimension or the FFD of $\mathbf{U}$, which can be determined as

$$
d_{\mathrm{fcm}}=\lim _{c \rightarrow c^{*}} \frac{\log F(c)}{\log c} .
$$

It can be further shown that if the plot of $\log F(c)$, the vertical axis, versus $\log c$, the horizontal axis, is represented by a straight line using the method of least squares, then a straight line in an $x-y$ diagram can be expressed as

$$
\log F(c)=d_{\mathrm{fcm}} \log c+b
$$

where $d_{\mathrm{fcm}}$ is the slope of the line and $b$ is the intercept of the fitting line with the vertical axis. Alternatively,

$$
F(c)=b c^{d_{\mathrm{fcm}}} .
$$

Thus, the FFD $d_{\mathrm{fcm}}$ expression in (9), in the limit where $c$ approaches $c^{*}$, can be determined as the slope of the plot of $\log F(c)$ versus $\log c$. The basic idea is that if an object is selfsimilar, then the slope of $\log F(c)$ versus $\log c$ is the same as the limit of $\log F(c) / \log c$ as $c$ approaches $c^{*}$. However, the determination of the slope is much easier than $c^{*}$.

Based on the minimization of $J_{m}$ expressed in (4), an infinite set of the FCM-based fractal dimensions $\mathcal{D}_{\text {fcm }}$ can be obtained in terms of the weighting exponent $m$ :

$$
\mathcal{D}_{\mathrm{fcm}}=\bigcup_{m=1}^{\infty} d_{\mathrm{fcm}}^{m}
$$

where a vector of finite or truncated FFDs can be obtained by setting the range for $m$ with some discrete values being greater than one (for $m=1$, the FCM algorithm becomes a hard-clustering case).

We now turn our discussion on the implementation of the FFD for pattern classification using VQ approach in the next section.

\section{Classification Using Vectorized FFD}

\section{A. Quantization of FFDs}

To obtain the set of codewords or codevectors, which can be modeled as the prototype of a certain class, we apply the method for quantizing the vectors of the FFDs. Let $\mathbf{a}_{t}$ be a vector of FFDs, and the codebook of the vectors of FFDs be $C=\left\{\mathbf{c}_{1}, \mathbf{c}_{2}, \ldots, \mathbf{c}_{L}\right\}$, where $\mathbf{c}_{j}=\left(c_{j 1}, c_{j 2}, \ldots, c_{j p}\right)$, $j=1,2, \ldots, L$, are codewords. Each codeword $\mathbf{c}_{j}$ is assigned to an encoding region $R_{j}$ in the partition $\left\{R_{1}, R_{2}, \ldots, R_{L}\right\}$. If the source vector $\mathbf{a}_{t}$ can be represented by the encoding region $R_{n}$, then its approximation, denoted as $V\left(\mathbf{a}_{t}\right)$, is

$$
V\left(\mathbf{a}_{t}\right)=\mathbf{c}_{n}, \quad \text { if } \mathbf{a}_{t} \in R_{n} .
$$

The main idea of the VQ [20] is to find an optimal codebook such that for a given training set and a codebook size, the average distortion in representing each vector $\mathbf{a}_{t}$ by the closest codeword $\mathbf{c}_{n}$ is minimum. One of the most popular methods for VQ design is the Linde, Buzo, and Gray (LBG) algorithm [21]. Given a training set $\mathbf{A}=\left\{\mathbf{a}_{1}, \ldots, \mathbf{a}_{N}\right\}$, a codebook of size $M^{*}$, and a splitting parameter $\epsilon>0$ which is a small real number, the LBG-VQ design algorithm is implemented by the following procedure.

1) Compute initial centroid: $M=1$, compute $\mathbf{v}_{M}=$ $\frac{1}{N} \sum_{n=1}^{N} \mathbf{a}_{n}$.

2) Splitting: For $i=1, \ldots, M$, split $\mathbf{v}_{i}$ into two vectors: $\mathbf{v}_{i}^{+}=\mathbf{v}_{i}(1+\epsilon)$ and $\mathbf{v}_{i}^{-}=\mathbf{v}_{i}(1-\epsilon)$; set $M=2 M$.

3) Updating: Apply the $c$-means algorithm to update the set of centroids (codewords) for the split codebook, $\left\{\mathbf{v}_{i}: i=\right.$ $1, \ldots, M\}$.

4) Iteration: Repeat steps 2 and 3 until the codebook of size $M^{*}$ is obtained.

\section{B. FFD-Based Classification}

Having briefly explained the concept and procedure of VQ, the classification system based on the fuzzy fractal analysis and VQ codebook approach works as follows. In the training phase, the samples are analyzed by the fuzzy fractal analysis to obtain the vectors of FFDs. The training FFD vectors are then quantized using the number of codebooks according to the number of different classes. In the testing phase, an input unknown sample, denoted as $s$, is analyzed by the fuzzy fractal analysis, which results in the FFD vector. The dissimilarity between the FFD vector of the unknown sample and each trained codebook is computed. The dissimilarity measure of the unknown sample $s$ and a particular known class represented by the codebook $C^{i}$ are determined using the minimum rule [22], [23]:

$$
D\left(s, C^{i}\right)=\min _{1 \leq j \leq L} D\left(\mathbf{a}, \mathbf{c}_{j}^{i}\right)
$$

where $D$ is a measure of dissimilarity taken as the $L_{2}$-norm, $\mathbf{a}$ is the FFD vector of the unknown sample $s\left(\mathbf{a}=\left\{d_{\mathrm{fcm}}^{k}\right\}\right.$, $k=1, \ldots, K$, where $K$ is the number of different values of $m$ defined in (3)), and $\mathbf{c}_{j}^{i}$ is the $j$ FFD-VQ codevector of a particular known class represented by codebook $C^{i}$.

The unknown sample $s$ is assigned to class $i^{*}$ if the dissimilarity measure of its FFD vector $\mathbf{a}$ and the FFD-VQ codebook 
$\mathbf{C}^{i}$ is minimum, i.e.,

$$
\begin{array}{r}
\operatorname{assign} s \text { to class } i^{*} \text { if } \\
i^{*}=\arg \min _{i} D\left(s, C^{i}\right) .
\end{array}
$$

In summary, the procedure of the FFD-based classification scheme works as follows.

\section{FFD-VQ-Based Classification}

1) Given a training set of object images, compute $\left\{d_{\mathrm{fcm}}^{k}\right\}$ according to (9) using various values of $m$ expressed in (3) for each image.

2) Given a codebook size, compute the codewords of the training set of $\left\{d_{\mathrm{fcm}}^{k}\right\}$ using a VQ method.

3) Given a test image, compute $\left\{d_{\mathrm{fcm}}^{k}\right\}$ according to (9) using the same various values of $m$ described in step 1 for the test image.

4) Assign the test image to the class, whose $\left\{d_{\mathrm{fcm}}^{k}\right\}$ is closest to the codebook of that class according to (15).

Application of the proposed approach for the analysis of a mouse mutant with brain morphological changes is presented and discussed in the following section.

\section{EXPERIMENT}

\section{A. Data}

All research described involving animals was approved by the Griffith University Animal Ethics Committee, and has complied with the guidelines and policies of the National Health, the Medical Council of Australia, and the Office of the Gene Technology Regulator. For tissue histology, mice were anesthetized with ketamine-xylazene $(4: 1)$ and perfused trancardially with $4 \%$ paraformaldehyde (PFA). Brains were removed and immersed in 4\% PFA overnight in vacuo. The following day brain tissue was dehydrated in a series of graded ethanol solutions $(50 \%, 70 \%, 100 \%)$ and then placed in dimethylsulfoxide for $60 \mathrm{~min}$ in vacuo. Tissue was then impregnated in a series of graded polyethylene glycol (PEG) solutions in vacuo and embedded in a mixture of PEG 1450/1000. Sagittal sections were cut on a rotary microtome at a thickness of $30 \mathrm{~m}$ and stained with 4',6-diamidino-2-phenylindole (DAPI). Cerebellum sections were viewed and captured using a Zeiss 1 fluorescence microscope and images were further processed with the digital image processing software AxioVision.

\section{B. Results}

Fig. 1(a) shows an image obtained from a section from a control (wild-type) mouse cerebellum, and Fig. 1(b) shows an image from a Pex13 brain mutant mouse cerebellum, both at postnatal day 10 (P10). Similarly, Fig. 2(a) and (b) shows the images of a section of control mouse cerebellum and mutant cerebellum, respectively, but at P15. To quantify the two phases of the control and the mutant brains of the mice, we applied the proposed framework of fuzzy scaling to estimate the FFDs of the two phases of the two groups. For the computation, we arbitrarily specified the fuzzy exponent $m=2.0-4.5$ with an

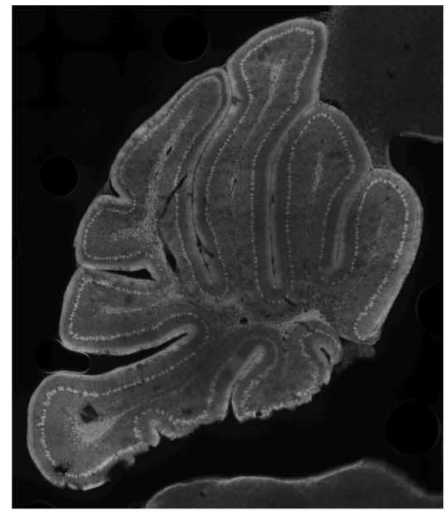

(a)

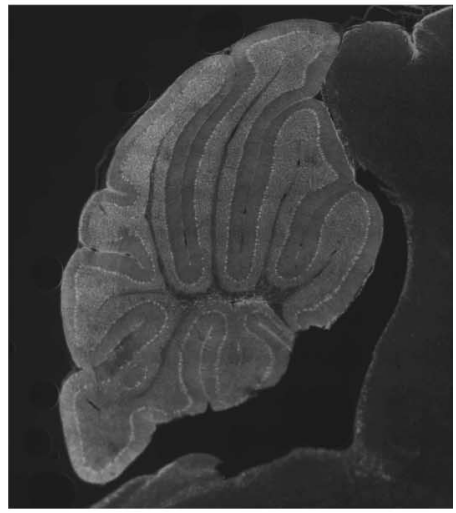

(b)

Fig. 1. Mouse cerebellum sections at P10: (a) control and (b) Pex13 brain mutant.

equal increment of 0.5 in this experimental study. In this study, the number of clusters were determined using (7). However, if $c^{*}$ is less than 8 , which is arbitrarily selected for line fitting, then eight clusters were used to fit the straight line for estimating $d_{\mathrm{fcm}}$. Because of the random initialization of the FCM that would not yield the same result for each invocation of the algorithm, for each fixed value of $m$, we generated ten different times for the FCM-based computation of the FFD of each model and selected the average value of $d_{\mathrm{fcm}}$.

Figs. 3 and 4 show the plots of the mean FFDs, $d_{\mathrm{fcm}}$, against $m$ of the control and mutant brains of the two phases at P10 and P15, respectively. These results were computed using the whole dataset. The standard deviations of the control and mutant at P10 are 0.0023 and 0.0030 , respectively, whereas the standard deviations of the control and mutant at P15 are 0.0035 and 0.0048 , respectively. The fractal dimensions obtained from the box-counting method [16], [24] of the P10 phase are 1.3461 for the control brain and 1.3987 for the mutant brain with the standard deviations of 0.0021 and 0.0028 for the control and mutant, respectively. Similarly, the box-counting-based fractal dimensions at P15 were estimated to be 1.3385 for the control brain and 1.3636 for the mutant brain with the standard deviations of 0.0026 and 0.0039 for the control and mutant, respectively. It is observed that the values of the fractal dimensions using the box-counting and FCM methods are below 2. For the box-counting method, it has been explained that this approach 


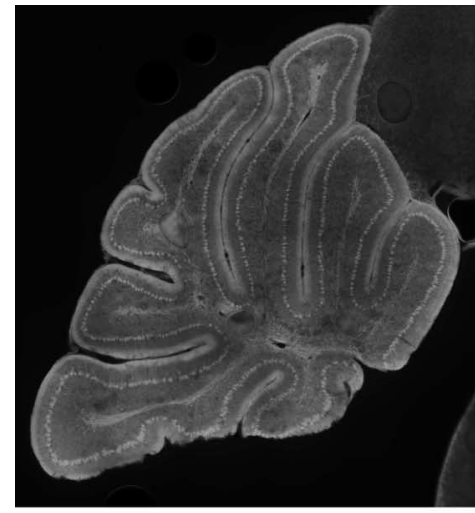

(a)

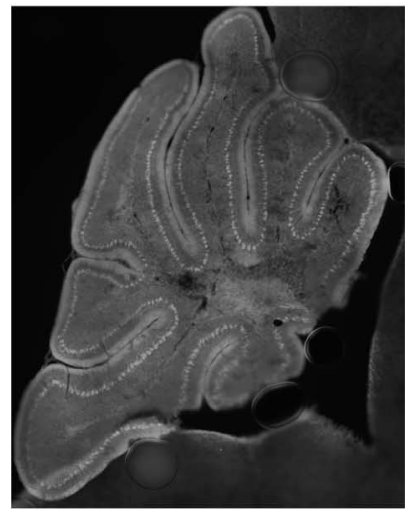

(b)

Fig. 2. Mouse cerebellum sections at P15: (a) control and (b) Pex13 brain mutant.

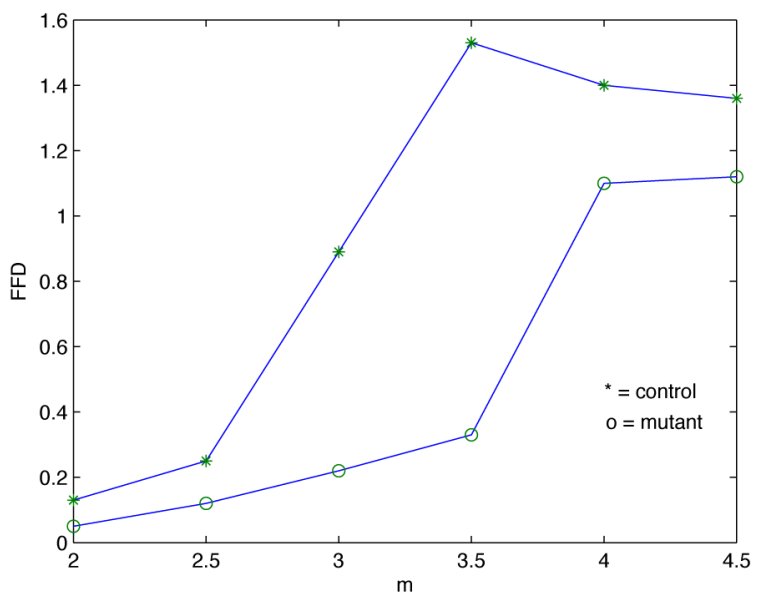

Fig. 3. FFDs of mouse cerebellum at P10.

used fractal techniques to evaluate the fitting parameters, but it cannot be directly associated with fractal geometry [11] - this is also in accordance with the findings of the work in [14] and [25]. The proposed FFD is likewise a function of the partition coefficients, and therefore, yields itself to the concept of fuzzy scaling rather than strictly to that of fractal geometry.

While the fractal dimensions estimated by the box-counting method show some difference between the control and mutant

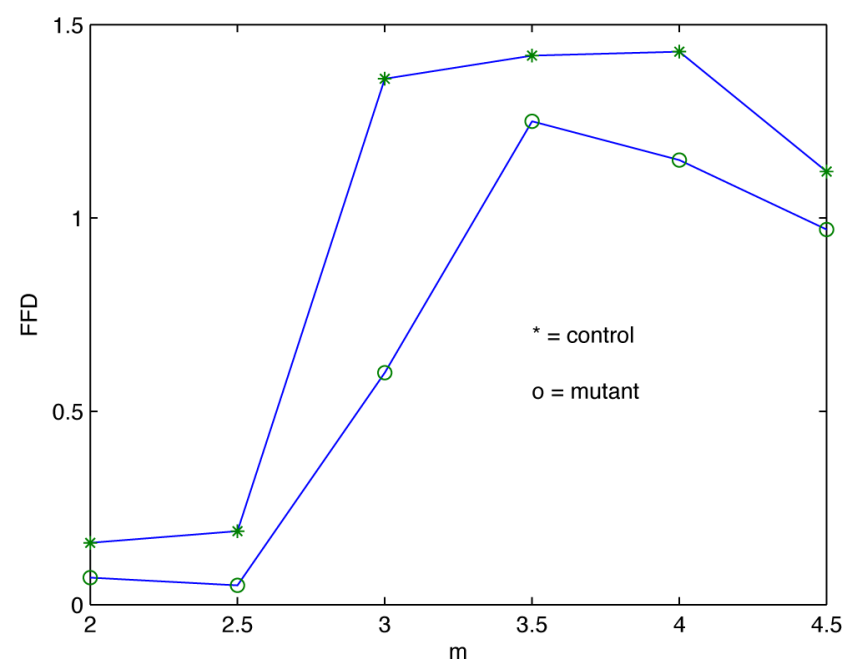

Fig. 4. FFDs of mouse cerebellum at P15.

brains, the plots of the FFDs in terms of the fuzzy exponent $m$ exhibit some distinct patterns for the different brains, where the FFDs tend to reach their maximum value about $m=3.5-4.0$. Interpretations of the differences in the FFDs between the control and mutant brains are as follows. There are changes in the foliation (folding) of the cerebellum. For example, the top left corner (north-west direction) of Fig. 2 shows a fold that is not present in the mutant brain. Figs. 1(a) and 2(a) show the gaps between different areas of the control brain in the lower left hand side, whereas in Figs. 1(b) and 2(b), the gaps are missing between these areas of the mutant brain, thus causing changes in the areas within the different parts of the brain. These effects indicate some morphological changes in the mutant brain. In addition, taking image intensitives into account by the formulation of the fuzzy objective function, the FFD values have the ability to capture this image feature to express the tissue differences between the two brain types. Using different values for the fuzzy exponent $m$, Figs. 3 and 4 further show that the FFDs vary with different values for $m$, and therefore, exhibit various numerical distinctions between the two models. For the P10 phase, the largest difference between the control and the mutant is when $m=3.5$, whereas for the P15 phase, when $m=3$. To verify the validity of the power law expressed in either (8) or (10) on which the proposed FFD is based, the cluster centers and the corresponding partition coefficients obtained from a mouse cerebellum at P10 were plotted to observe the linearity of the data points. As a result, Fig. 5 shows the values of $\log c$ versus $\log F(c)$, where a straight line was fitted in a least-squares sense. It can be seen that the straight line fits closely to the data points, indicating the valid assumption of the power law described in either (8) or (11).

Specificity (SP) in diagnosis is a critical measure of the costeffective design of clinical classification methods. When referring to a medical test, the SP is the probability that the test will be negative among patients who do not have the disease. While SP is the percentage of the control (nondiseased) samples that are correctly identified, sensitivity (SE) is the percentage of the diseased (mutant) samples that are correctly identified. 


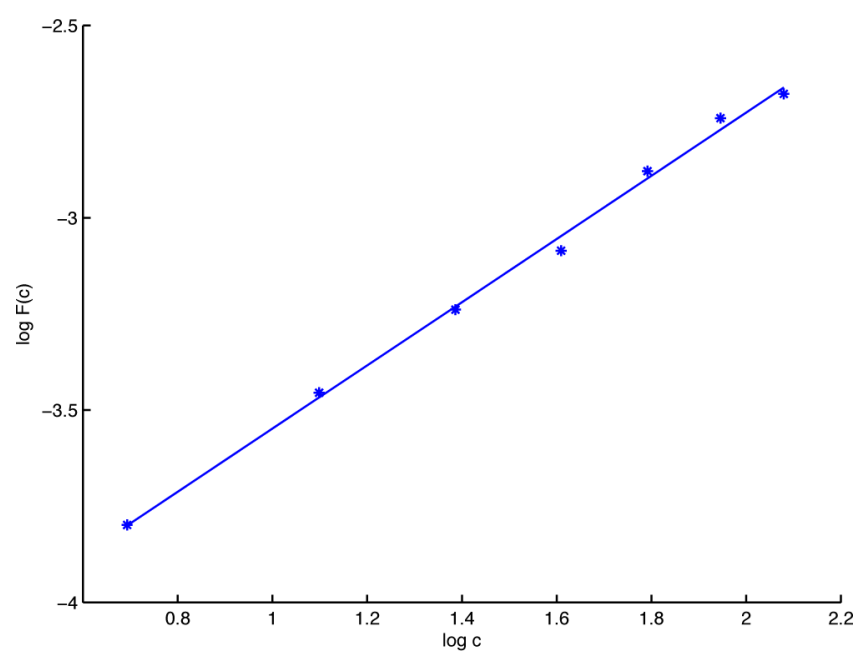

Fig. 5. FFD taken as the slope of the straight line.

TABLE I

SP RESUlts Obtained From Various Fractal Methods

\begin{tabular}{lcccccc}
\hline Method & \multicolumn{3}{c}{ Phase 1 } & \multicolumn{3}{c}{ Phase 2 } \\
\hline & 8 & 16 & 32 & 8 & 16 & 32 \\
\hline BFD & 65.27 & 66.32 & 66.45 & 66.31 & 66.52 & 66.58 \\
PFD & 66.15 & 66.22 & 66.37 & 66.26 & 66.70 & 66.84 \\
IFFD1 & 72.52 & 73.61 & 73.90 & 74.29 & 74.85 & 75.01 \\
IFFD2 & 72.89 & 73.57 & 73.80 & 74.67 & 74.89 & 75.26 \\
IFFD3 & 85.23 & 87.61 & 88.69 & 86.50 & 87.66 & 88.27 \\
IFFD4 & 85.56 & 87.46 & 88.91 & 86.92 & 87.88 & 88.52 \\
IFFD5 & 78.00 & 78.64 & 79.02 & 78.59 & 79.41 & 79.89 \\
IFFD6 & 77.81 & 78.20 & 78.71 & 78.04 & 78.77 & 79.00 \\
VFFD & 88.47 & 89.50 & 90.16 & 89.00 & 89.64 & 90.07 \\
\hline
\end{tabular}

SP and SE are mathematically defined as [26] SP $=\mathrm{TN} /(\mathrm{TN}$ + FP), where TN and FP are the numbers of true negative and false positive, respectively, and $\mathrm{SE}=\mathrm{TP} /(\mathrm{TP}+\mathrm{FN})$, where $\mathrm{TP}$ and $\mathrm{FN}$ are the numbers of true positives and false negatives, respectively. In this study, the sum of TN and FP, and the sum of TP and FN are equivalent to the total numbers of the control and mutant samples, respectively. Out of the total of 11 samples for each of P10 control and mutant mice, and 24 samples for each of P15 control and mutant mice, we equally divided each population of the samples for training and testing using the vectorized FFD (VFFD) based classification approach. For each sample, we generated six FFDs using $m=2.0-4.5$ with an equal increment of 0.5 . We used the codebook sizes of 8,16 , and 32 for training the samples of the two phases. In addition to the use of the box fractal dimensions (BFDs) for comparison with the proposed method, we also estimated the fractal dimensions of the samples using the power spectrum method [27] where the fractal dimension can be determined from the Hurst exponent. We applied the same classification design and principle, but these were based on scalar quantization for BFDs and the power-spectrum-based fractal dimensions (PFDs).

The SP probabilities obtained from VFFD-based, BFD-based, and PFD-based classifications of the two phases are shown in Table I, where phases 1 and 2 indicate P10 and P15, respectively, and 8,16 , and 32 are the codebook sizes. Table II shows the SE probabilities obtained from both FFD-based and BFDbased classifications of the two phases. The results on SE for
TABLE II

SE Results ObTaIned From Various Fractal Methods

\begin{tabular}{lcccccc}
\hline Method & \multicolumn{3}{c}{ Phase 1 } & \multicolumn{3}{c}{ Phase 2 } \\
\hline & 8 & 16 & 32 & 8 & 16 & 32 \\
\hline BFD & 57.14 & 59.70 & 60.11 & 60.88 & 61.34 & 61.75 \\
PFD & 58.67 & 59.81 & 60.47 & 61.11 & 61.45 & 61.86 \\
IFFD1 & 65.30 & 66.61 & 67.22 & 66.28 & 67.19 & 67.52 \\
IFFD2 & 65.19 & 66.20 & 66.80 & 66.58 & 67.06 & 67.50 \\
IFFD3 & 69.30 & 69.82 & 70.49 & 70.27 & 71.32 & 72.09 \\
IFFD4 & 70.56 & 71.83 & 72.17 & 71.08 & 72.17 & 73.29 \\
IFFD5 & 69.06 & 69.47 & 70.42 & 70.19 & 70.24 & 70.48 \\
IFFD6 & 69.27 & 69.80 & 70.65 & 70.26 & 70.39 & 70.51 \\
VFFD & 71.82 & 73.41 & 74.22 & 73.09 & 74.66 & 75.56 \\
\hline
\end{tabular}

both BFD-based and VFFD-based are lower that those on SP. This can be explained in that there is more variability of the mutant patterns than that of the control patterns, which lead to a lower classification rate for the mutant samples. To investigate the performance of individual FFDs (IFFDs) on the classification, the IFFDs were also applied to obtain the SP and SE measures of the samples. The results on SP and SE given by the IFFD are also shown in Tables I and II, respectively, where IFFD $1, \ldots$, IFFD6 stand for the FFD numbers among the set of six FFDs. While IFFDs outperform both the BFD and the PFD, the vectorized FFD-based method outperforms all other methods and particularly improves the classification rates over the IFFD. In general, both SP and SE results presented in Tables I and II indicate the superior performance of the proposed approach.

To obtain numerical evidence against the claims of the results on the SP and SE using the proposed vectorized FFD-based method, we randomly selected other 25 training and testing datasets to carry out the classifications and applied the $z$-tests, where a 5\% significance level is adopted. The $p$-values obtained from the $z$-tests, which are the probabilities of observing the given results if the assertions are true (small values of $p$ cast doubt on the validity of the assertions), for the codebook sizes of 8,16 , and 32 are $0.6029,0.6274$, and 0.6310 , respectively. The results of the hypothesis tests indicate that the average performance data are statistically significant at the 5\% significance level.

\section{CONCLUSION}

We have presented in this study an effective method for characterizing changes to cerebellum morphology in a Pex13deficient mouse mutant. The fuzzy scaling in the brain imaging of these mouse mutants appears to offer a promising application tool for describing the complex features of tissue pathology associated with this disease. The innovative concept of fuzzy fractals, which scale to infinity, is hoped to lead to some mechanistic explanations of other related complex biological processes-in particular, the biological implications of the phase space analysis in fractals and chaos to determine the pattern of the mechanism underlining the generated data.

There are some important issues for further investigations of the properties of the proposed fuzzy scaling. These include the optimal selection of the interval and the range of the fuzzy exponent $m$, the optimal alternative selection of the number of clusters in case these become too small using (7), and the 
possible use of feature selection of the FFDs for classification improvement. Another potential issue is to incorporate the fuzzy objective function with other statistical or spatial properties in order to capture appropriate features of different objects of interest. Nevertheless, the proposed conceptual framework can be useful for the analysis of other problems in the life sciences.

\section{REFERENCES}

[1] G. A. Losa, G. Baumann, and T. H. F. Nonnenmacher, "Fractal dimension of pericellular membranes in human lymphocytes and lymphoblastic leukemia cells," Pathol. Res. Pract., vol. 188, pp. 680-686, 1992.

[2] S. J. Gould, D. Valle, and G. V. Raymond, "The peroxisome biogenesis disorders," in The Metabolic and Molecular Bases of Inherited Disease, C. R. Scriver, A. L. Beaudet, W. S. Sly, and D. Valle, Eds. New York: McGraw-Hill, 2001.

[3] H. W. Moser, "Peroxisomal disorders: Classification and overview of biochemical abnormalities," Rev. Neurol., vol. 28, no. 1, pp. S45-S48, 1999.

[4] Y. Liu, J. Bjorkman, A. Urquhart, R. J. Wanders, D. I. Crane, and S. J. Gould, "PEX13 is mutated in complementation group 13 of the peroxisome biogenesis disorders," Amer. J. Hum. Genet., vol. 65, pp. 621-634, 1999.

[5] A. Nagy, "Cre recombinase: The universal reagent for genome tailoring," Genesis, vol. 26, pp. 99-109, 2000.

[6] N. Shimozawa, Y. Suzuki, Z. Zhang, A. Imamura, R. Toyama, S. Mukai, Y. Fujiki, T. Tsukamoto, T. Osumi, T. Orii, R. J. Wanders, and N. Kondo, "Nonsense and temperature-sensitive mutations in PEX13 are the cause of complementation group $\mathrm{H}$ of peroxisome biogenesis disorders," Hum. Mol. Genet., vol. 8, pp. 1077-1083, 1999.

[7] M. Maxwell, J. Bjorkman, T. Nguyen, P. Sharp, J. Finnie, C. Paterson, I. Tonks, B. C. Paton, G. F. Kay, and D. I. Crane, "Pex13 inactivation in the mouse disrupts peroxisome biogenesis and leads to a Zellweger syndrome phenotype," Mol. Cell. Biol., vol. 23, pp. 5947-5957, 2003.

[8] G. Landini, "Applications of fractal geometry pathology," in Fractal Geometry in Biological Systems-An Analytical Approach, P. M. Iannaccone and M. Khokha, Eds. Boca Raton, FL: CRC Press, 1996.

[9] R. Sedivy, Ch. Windischberger, K. Svozil, E. Moser, and G. Breitenecker, "Fractal analysis: An objective method for identifying atypical nuclei in dysplastic lesions of the cervix uteri," Gynecol. Oncol., vol. 75, pp. 78-83, 1999.

[10] F. Bernard, J. L. Bossu, and S. Gaillard, "Identification of living oligodendrocyte developmental stages by fractal analysis of cell morphology," $J$. Neurosci. Res., vol. 65, pp. 439-445, 2001.

[11] D. Ristanovic, V. Nedeljkov, B. D. Stefanovic, N. T. Milosevic, M. Grgurevic, and V. Stulic, "Fractal and nonfractal analysis of cell images: Comparison and application to neuronal dendritic arborization," Biol. Cybern., vol. 87, pp. 278-288, 2002.

[12] J. W. Baish and R. K. Jain, "Fractals and cancer," Cancer Res., vol. 60, pp. 3683-2688, 2000.

[13] J. C. Anderson, A. L. Babb, and M. P. Hlastala, "A fractal analysis of the radial distribution of bronchial capillaries around large airways," J. Appl. Physiol., vol. 98, pp. 850-855, 2005.

[14] J. W. Fuseler, C. F. Millette, J. M. Davis, and W. Carver, "Fractal and image analysis of morphological changes in the actin cytoskeleton of neonatal cardiac fibroblasts in response to mechanical stretch," Microsc. Microanal., vol. 13, pp. 133-143, 2007.

[15] B. B. Mandelbrot, "How long is the coast of Britain? Statistical selfsimilarity and fractional dimension," Science, vol. 156, pp. 636-638, 1967.

[16] L. S. Liebovitch, Fractals and Chaos Simplified for the Life Sciences. New York: Oxford Univ. Press, 1998.

[17] H. M. Hastings and G. Sugihara, Fractals: A User's Guide for the Natural Sciences. New York: Oxford Univ. Press, 1993.

[18] S. Lovejoy, "Area-perimeter relation for rain and cloud areas," Science, vol. 216, pp. 185-187, 1982
[19] J. C. Bezdek, Pattern Recognition With Fuzzy Objective Function Algorithms. New York: Plenum, 1981.

[20] R. M. Gray, "Vector quantization," IEEE Acoust., Speech, Signal Process. Mag., vol. 1, no. 2, pp. 4-29, Apr. 1984.

[21] Y. Linde, A. Buzo, and R. M. Gray, "An algorithm for vector quantization," IEEE Trans. Commun., vol. 28, no. 1, pp. 84-95, Jan. 1980.

[22] D. O'Shaughnessy, Speech Communication-Human and Machine. Reading, MA: Addison-Wesley, 1987.

[23] L. Rabiner and B. H. Juang, Fundamentals of Speech Recognition. Englewood Cliffls, NJ: Prentice-Hall, 1993.

[24] L. S. Liebovitch and T. I. Tóth, "A fast algorithm to determine fractal dimensions by box counting," Phys. Lett. A, vol. 141, pp. 386-390, 1989.

[25] J. Panico and P. Sterling, "Retinal neurons and vessels are not fractal but space-filling," J. Comp. Neurol., vol. 361, pp. 479-490, 1995.

[26] I. Levner. (2005). Feature selection and nearest centroid classification for protein mass spectrometry. BMC Bioinf. [Online]. vol. 6, p. 68. Available: http://www.biomedcentral.com/1471-2105/6/68

[27] H. O. Peitgen, H. Jürgens, and D. Saupe, Chaos and Fractals: New Frontiers of Science. New York: Springer-Verlag, 1992.

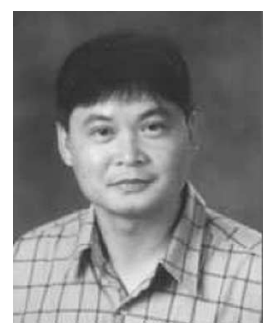

Tuan D. Pham (M'95-SM'01) received the Ph.D. degree in civil engineering from the University of New South Wales, Sydney, Australia, in 1995.

$\mathrm{He}$ is an Associate Professor in the School of Information Technology and Electrical Engineering, University of New South Wales. His current research interests include image processing, pattern recognition, fuzzy-set algorithms, geostatistics, bioinformatics, and biomedical informatics.

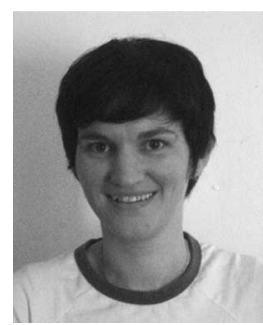

Catharina C. Müller received the Diploma in biology from the University of Konstanz, Konstanz, Germany. She is currently working toward the Ph.D. degree at Griffith University, Nathan, Qld., Australia.

She was with Max Delbruck Center for Molecular Medicine, Berlin. Her current research interests include the characterization of a conditional mouse model for Zellweger Syndrome.

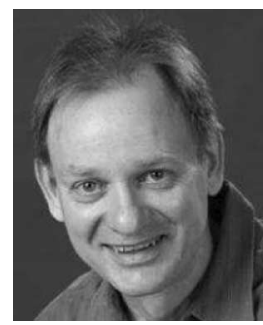

Denis I. Crane received the Ph.D. degree from Griffith University, Nathan, Qld., Australia.

During 1989, he was appointed as a Lecturer at Griffith University, where he has been a Professor since 2008. His research fields include molecular cell biology, biochemistry, and human genetics. His current research interests include the characterization of mouse models of Zellweger syndrome, the molecular basis of neurodegeneration of this and other neurodegenerative disorders, and mechanisms involved in the import of proteins into peroxisomes. 